\title{
Cumulative effects of transgenerational induction on plant palatability to generalist and specialist herbivores
}

\author{
Isabelle P. Neylan ${ }^{1,2}$, Rodolfo Dirzo ${ }^{1}$, and Mar Sobral ${ }^{1,3,4}$ \\ ${ }^{1}$ Department of Biology, Stanford University, Stanford, CA 94305, USA \\ ${ }^{2}$ Department of Ecology and Evolution, University of California, Davis, CA 95616, USA \\ ${ }^{3}$ CEFE, University Paul Valéry Montpellier 3, University Montpellier, EPHE, CNRS, IRD, Montpellier, France \\ ${ }^{4}$ Departmento de Zooloxía Xenética e Antropoloxía Física, Universidade de Santiago de Compostela, \\ Santiago de Compostela, La Coruña, Spain \\ Correspondence: Isabelle P. Neylan (ineylan@gmail.com)
}

Received: 8 January 2018 - Revised: 30 January 2018 - Accepted: 31 January 2018 - Published: 16 March 2018

\begin{abstract}
Herbivore damage can induce anti-herbivore traits in plants. However, there is little data regarding how these induced traits affect a plant's palatability (an important factor in determining the likelihood and magnitude of herbivore damage) across multiple generations post-induction, or whether the effect of transgenerational induction differs between generalist and specialist herbivores. Here we used palatability as a measure of the effects of transgenerational defensive induction in wild radish plants. We conducted a greenhouse experiment to determine whether generalist (slugs) and specialist (caterpillars of the white cabbage butterfly) herbivores' preference for wild radish differed depending on the number of previous generations that experienced herbivory. We found lowered palatability in plants with two or three inductions in their past in the case of generalist slugs, while palatability to a specialist herbivore was not affected by transgenerational induction. We conclude that the history of herbivory experienced by a plant's ancestors over multiple generations may play an important role in its ability to defend itself against generalist herbivores, but not against the specialists with whom they have co-evolved. Our findings suggest that the effects that multiple past inductions may have on palatability down the family line can be expected to have ecological and evolutionary implications.
\end{abstract}

\section{Introduction}

Defense against herbivory is recognized as one of the main factors shaping evolutionary trajectories of plants and their herbivores (Ehrlich and Raven, 1964; Heil, 2008; Thompson and Burdon, 1992; Strauss and Agrawal, 1999). The induction of plant defenses as a response to herbivory has been shown to affect herbivore performance, and to affect the palatability of a plant during future instances of herbivory in its lifetime (Agrawal, 1999, 2000; Brian Traw and Dawson, 2002), and for one subsequent generation (Agrawal et al., 1999a). Evidence is also accumulating that transgenerational effects may potentially last multiple generations (Holeski et al., 2012; Rasmann et al., 2012). However, it has not yet been examined whether there are measurable conse- quences, in terms of palatability, of having multiple generations of ancestors that experienced herbivory and whether these consequences differ between specialist and generalist herbivores.

While there are costs associated with induction of defenses (Agrawal et al., 1999b; Heil, 2002; Cipollini et al., 2003; Zangerl, 2003), plants with lowered palatability will likely have less tissue consumed by herbivores and thus be able to allocate more resources to growth and reproduction (Simms and Rausher, 1987; Agrawal, 1998; Strauss and Agrawal, 1999). Through a mechanism such as heritable epigenetic changes, the benefits of having induced defenses may be passed along to offspring potentially priming the defenses of future generations (Jablonka and Lamb, 1998; Takeda and Paszkowski, 2006; Richards, 2006; Verhoeven et al., 2010). 
Therefore, the effect that multiple past inductions may have on palatability down the family line can be expected to have ecological and even evolutionary implications.

To examine the effects of defensive induction over multiple generations, we conducted an experiment involving multiple generations of wild radish plants (Raphanus savitus). Previous studies have also shown that specialist and generalist herbivores (those that have evolved to feed on a specific species or family of plants, and those that can eat a wide variety of plant taxa, respectively) may differ in their reactions to plant defenses (Agrawal, 2000; Cornell and Hawkins, 2003; Ali and Agrawal, 2012). Therefore, we used both specialist (Pieris rapae caterpillars) and generalist (the terrestrial slug Deroceras reticulatum) herbivores of this plant to illuminate any potential differences. We examined whether the experiences of ancestors influence the defense capabilities of a plant against herbivores, and the possible ecological implications thereof in terms of plant palatability. Specifically, we asked: are there differences in plant palatability to specialist and generalist herbivores depending on the number $(1,2$, or 3) of generations that experienced herbivory?

\section{Materials and methods}

\subsection{Plants}

Wild radish (Raphanus sativus) plants are fast-growing with short generation times and are abundant in open, disturbed areas throughout the United States and on every continent except Antarctica (Holm, 1997). In the field, they are attacked by both specialist herbivores (caterpillars of the white cabbage butterfly, Pieris rapae), as well as a multitude of generalist herbivores, including many insects and terrestrial mollusks (Karban and Nagasaka, 2004). The plant's inducible defenses include toxic secondary metabolites (glucosinolates) that are used to ward off herbivores (Agrawal, 1999) and hairs (trichomes) that serve as a physical deterrent against herbivores feeding on the leaves (Agrawal, 1999).

The wild radish plants used in this experiment were the fourth generation of those grown under controlled and standardized greenhouse conditions at the Stock Farm Plant Growth Facility, Stanford University, Stanford, CA. The induction history (whether or not they were exposed to herbivores) of the plants' parents and grandparents is known, and within these generations and the current generation there was the potential for up to three generations to have experienced attack, and therefore induction by herbivores. Plants were induced, reared, and maintained in the same controlled conditions in each generation. The seeds were germinated and the established plants maintained in the greenhouse. Plants were initially planted in germination flats and then transferred to $0.8 \mathrm{~L}$ pots after about two weeks. The soil mixture consisted of $75 \%$ peat moss (Orchard Supply Hardware, San Jose, CA, USA), $20 \%$ potting soil, and $5 \%$ fine white sand. Plants were watered once a day and were maintained at 26 and $21^{\circ} \mathrm{C}$ in
$12 \mathrm{~h}$ cycles. Leaf samples were taken from plants late into their two-leaf stage at about one month old.

The first generation $\left(\mathrm{F}_{0}\right)$ was not induced and represented a single maternal family. This reduced the initial amount of genetic variability, allowing us to detect plasticity effects given the uniform genetic background. We subsequently raised three more generations. In all generations, we induced half of the plants by exposing them to natural herbivory from the specialist caterpillar to Brassicacae, P. rapae, and kept the other half non-induced. In our experiment, forty-four plants of the $\mathrm{F}_{3}$ generation were used with induction histories as follows: INN, NIN, NNI, IIN, INI, NII, and III (I = induced, $\mathrm{N}=$ non-induced). The induction history included treatment of the plant's grandparents, treatment of the plant's parents, and treatment of the experimental plant itself, in this order. (For example, NNI would mean non-induced grandparents, non-induced parents, and induced current plant.) This design allowed us to investigate potential intra- vs. intergenerational effects. Each of the 44 plants was used in three palatability trials for a total of 132 replicated assays, 66 with caterpillars and 66 with slugs. Each induction history was represented by 5 to 8 plants belonging to three groups representing plants with one, two, and three past inductions.

\subsection{Caterpillars}

The white cabbage butterfly caterpillars ( $P$. rapae) are known to be specialized herbivores to the Brassicaceae family, which includes wild radish. The caterpillars were purchased and shipped from Carolina Biological Company (CBC). The caterpillars were kept in small plastic containers provided by CBC with five to six larvae per container and an agar and wheat-based food medium was provided ad libitum. Caterpillars arrived during their second or third instar and were used in the experiments immediately upon arrival. The same twenty-two caterpillars were used for all three trials.

\subsection{Slugs}

Common European garden slugs (Deroceras reticulatum) are known to feed on many species of plants from disturbed areas and therefore represent a good candidate to assess palatability in a comparative way (Dirzo, 1980). Although a non-native species in California, D. reticulatum is typically present in areas where wild radish is common and has been seen feeding on this plant (Rodolfo Dirzo, personal observations, 2018). The slugs were collected in the field over a three-week period from the area surrounding the Stanford University Plant Growth Facility and from gardens in Palo Alto, CA. The slugs were brought back to the lab and kept individually in Tupperware containers modified with air holes and a damp cotton ball. The slugs were fed an all lettuce diet for at least two weeks prior to the palatability trials. Twentytwo slugs of comparable size were used for the palatability trials. 


\subsection{Palatability tests}

For the palatability trials, leaf tissue was collected from radish plants and placed in a petri dish with either a slug or a caterpillar. Using a circular 1-inch diameter corer to ensure uniform size and leaf edge, leaf tissue was cut from the newest leaves of the corresponding plants in the greenhouse and placed into labeled test tubes. Leaf samples were brought to the lab immediately after harvesting. To avoid desiccation of the leaf circles, the tubes (with the lids tightly sealed) were placed in a water bath. Fresh tissue pieces were collected before each trial for a total of three trials, with a different plant-animal pair each time. Each leaf circle was weighed using a microbalance to obtain an initial weight before each trial. With the exception of trial one, the area of each leaf piece (or remaining parts thereof) was also photographed with a reference ruler and analyzed throughout the trials using ImageJ software and a protocol similar to that of O'Neal et al. (2002).

To run the trials, individually identified slugs and caterpillars were assigned to individual petri dishes to keep track of plant-animal pairs during the palatability test. Each animal was weighed and placed in its dish with a wet cotton ball to avoid desiccation, and presented with one leaf disc per trial. The weight (all trials) and area (trials two and three) of the leaves were measured after being left over night $(18 \mathrm{~h})$. In addition, leaf pieces from several different individuals were also left in the same manner but without an animal present during all three trials to control for possible weight and area lost due to desiccation. The remaining leaf tissue was then removed while the animals remained in their labeled petri dishes until the next trial, ensuring a new animal and plant pair each time. Foliage from each individual plant was exposed at least once to both a slug and a caterpillar. All three trials began and ended at the same time of day for three consecutive days.

\subsection{Statistical analyses}

Leaf weight consumed overnight was analyzed using general linear models in which the lab trial, animal type (slug or caterpillar), number of inductions, and accumulated treatment across generations (nested in number of inductions) as well as the interactions between animal type and treatment nested in number of inductions were included as fixed factors. Accumulated treatment across generations is a factor that accounts for the fact that besides the number of ancestral inductions, the order in which they occurred may also be important. For example, a plant with a history of induction of IIN will be different than one with NII or INI even if all would have a value of 2 for the number of accumulated inductions across generations. Animal weight was first included in the models, but it was not significant and did not help the model fit, so it was later excluded. The leaf area consumed showed similar patterns. We analyzed the weight con-

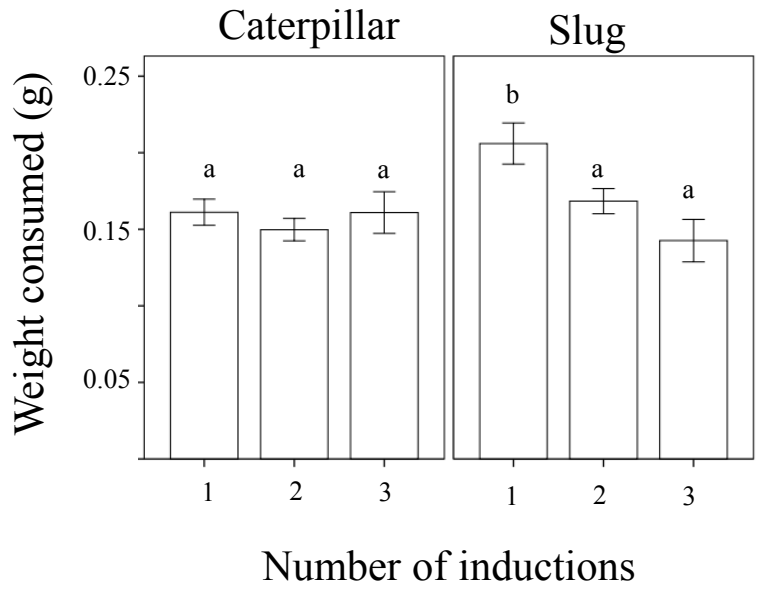

Figure 1. Weight of leaf tissue consumed overnight of wild radish plants with one, two, or three generations of induction driven by exposure to herbivory in the plants' family's history for both caterpillars and slugs.

sumed of leaf tissue from 44 plants by 44 animals ( 22 caterpillars and 22 slugs) in 3 different trials $(n=132)$. There were seven different treatment levels (NNN, NIN, NNI, IIN, INI, NII, and III), for which we had between five and eight plants tested three times each.

\section{Results}

The amount of plant tissue consumed was significantly affected by animal type (caterpillar or slug), the total number of inductions in the plant's ancestry $(1,2$, or 3 generations of past induction), and the induction history across generations (the order of inductions or non-inductions) (Table 1). In general, the generalist herbivore (slugs) ate more leaf material than the more specialized caterpillars (Fig. 1). Additionally, a marginally significant interaction $(p=0.051)$ was detected between animal type and the treatment with the effect of treatment in palatability being larger in slugs than in caterpillars. While the number of inductions did not appear to affect the palatability of the plants when caterpillars were the test herbivore, plants with 2 or 3 instances of induction in their past were significantly less palatable to slugs than those with only one instance of induction (Fig. 1). The induction history across generations was also significant in the model (Table 1, Fig. 2). Between plants with one generation of induction, slugs found less palatable those plants that had been exposed to herbivores themselves (NNI) than those plants with their parents or grandparents exposed (NIN and INN, respectively), thus implying a greater decrease in palatability to generalists due to current herbivory conditions than to herbivory across generations. 
Table 1. Results of the general linear model used to examine the effects of induction on wild radish palatability.

\begin{tabular}{lrrr}
\hline & df & $F$ & $P$ \\
\hline Lab trial & 2116 & 33.763 & $<0.001$ \\
Animal type & 1116 & 5.363 & 0.022 \\
Number of inductions & 2116 & 4.260 & 0.016 \\
Across generations treatment (number of inductions) & 4116 & 6.386 & $<0.001$ \\
Animal type $\times$ treatment (number of inductions) & 6116 & 2.163 & 0.051 \\
\hline
\end{tabular}

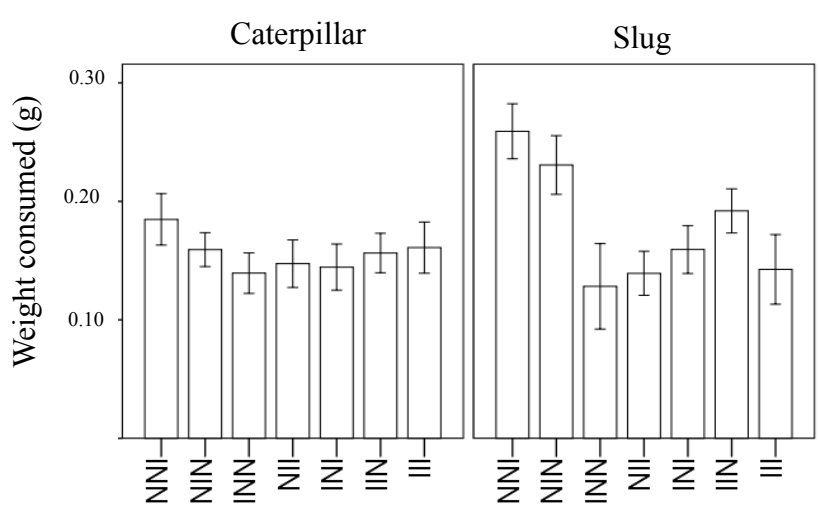

Treatment

Figure 2. Weight of leaf tissue of wild radish plants consumed overnight, depending on history of induction ( $\mathrm{I}=$ induced, $\mathrm{N}=$ noninduced). The treatment code starts with the grandmaternal induction treatment, followed by the maternal induction treatment, and ending with the treatment for the current plant.For example, the plant furthest to the left would have induced grandparents, noninduced parents, and be non-induced in its current generation.

\section{Discussion}

We found that plants with two or three inductions in their past manifested a decrease in palatability when exposed to a generalist herbivore, but not when exposed to a specialist herbivore. Thus, herbivory experienced by previous plant generations influenced the palatability of their offspring, presumably because of accumulated changes in defensive plant traits. We show that herbivory experienced during several generations has cumulative, ecologically significant consequences affecting the current interaction with generalist herbivores.

From a long-term survival perspective, this is a potentially meaningful result. The plants' ability to pass on useful traits based on their surroundings is beneficial if their offspring will be experiencing similar herbivory conditions. If the environment is variable, perhaps because the abundance of herbivores oscillates considerably, then having offspring inherently allocating resources in unnecessary defenses could be detrimental (Harvell, 1990; Agrawal et al., 1999b; Karban et al., 1999; Karban and Nagasaka, 2004). Our results suggest that by being more conservative in transgenerational influ- ences, the plants may ensure that only changes that will be beneficial to their offspring are passed down. There may be a greater chance that a plant will experience herbivory if the past two or three generations have all experienced it, than if only one generation has. This finding is further supported by theory concerning non-genetic inheritance and its role in evolution, namely that transgenerational responses should be adaptive in environments that are variable but predictable from the parental environment (Uller, 2008; Bonduriansky et al., 2012). Alternatively, multiple generations of exposure may be necessary to produce an effect strong enough to be detected or to "erase" the effects of inconsistent environmental cues in the past (Holeski et al., 2012).

We also found a potential difference in the size of the effect of induction intra-generation vs. inter-generation. When presented to slugs, plants that were themselves induced but lacked parental or grandparental induction (NNI) had lower palatability than those with either parental or grandparental induction but no herbivore exposure in their own generation (INN or NIN). Intra-generational induction seems to have a stronger effect on palatability than inter-generational induction. A similar result was found when looking at maternal effects of herbivore induction in parents and offspring of Raphanus raphanistrum (Agrawal, 2002).

Our results showed interesting differences between the generalist and specialist herbivores. While generalist slugs ate more leaf material overall (likely due to their size), the effects of induction on palatability were greater in slugs than in caterpillars, with caterpillars not affected by a plants' ancestral inductions. This is potentially related to the close coevolution between specialist cabbage caterpillars and Brassicaceae, in which the latter would have evolved to better handle the defenses of their target plants (Meijden, 1996; Agrawal, 2000; Karban and Nagasaka, 2004; Bossdorf et al., 2004).

There are many unknowns in the so called "specialistgeneralist paradigm" as to how both the herbivores and the plants they are feeding on respond to each other, namely because each is affected by the other (Ali and Agrawal, 2012). For example, there is evidence that generalists can elicit a different defensive response from a plant than a specialist (Agrawal, 2000). In our experiment this fact was controlled for with all plants being induced by specialist caterpillars. Previous studies have found similar plant defense responses 
to both specialist and generalist herbivores in the Brassicaceae family (Reymond et al., 2000; Poelman et al., 2008). Our results are consistent with others that show that specialists are less affected by their target plant's defense compounds than generalists at low levels of defense compound (Agrawal and Kurashige, 2003). However, generalists have also been shown to handle plant defenses better than specialists, allowing them to eat a wider range of species (Ali and Agrawal, 2012). Nevertheless we found that even though slugs found the radish foliage to be acceptable, they still consistently preferred plant tissue that had experienced fewer transgenerational inductions.

Agrawal et al. (1999b) showed that wild radish plants with parents that experienced induction had heightened defense phenotypes (higher concentrations of glucosinolates and higher densities of trichomes), which led to a lowered performance of the specialist $P$. rapae caterpillars. Our results demonstrate a correspondence between past cumulative inductions and lowered palatability to generalist herbivores and highlight potential differences between ecological consequences for specialist and generalist interactions. We found that the number of inductions in a plant's ancestry dictated their palatability to slugs. Thus, while the experience of a plant itself had the potential to reduce palatability (intra-generational plasticity), the experiences of their parents and grandparents also play a role cumulatively (intergenerational plasticity).

Recent studies have shown that epigenetic modifications are often responsible for transgenerational phenotypic changes (Jablonka and Lamb, 1998; Takeda and Paszkowski, 2006; Richards, 2006; Verhoeven et al., 2010). Our study is relevant in this context given the transgenerational effects on palatability we detected and its consequences for plantherbivore interactions and in light of the fact that induced plant defenses have been found to be epigenetically heritable (Rasmann et al., 2012). Epigenetics, broadly defined as a heritable change in the expression of genes that is not explained by a change in the DNA nucleotidic sequence (Bossdorf et al., 2008), has become an enticing field of study because it illuminates how the environment directly affects gene expression (Agrawal, 2001; Galloway and Etterson, 2007).

For epigenetics to have a real effect on organisms and their evolution in nature, it must have ecological implications that translate into a concrete difference in fitness (Bossdorf et al., 2008). We show that transgenerational defenses, which may be related to epigenetic effects, do have important ecological benefits in the form of reduced palatability to generalists but not to specialist herbivores. Our findings support the idea that transgenerational changes like the ones we uncovered have ecological consequences with important evolutionary implications. The strikingly contrasting response of the two herbivores is an aspect that warrants further examination.
Data availability. Raw data accessible at https://doi.org/10.6084/m9.figshare.5956666.v1.

Competing interests. The authors declare that they have no conflict of interest.

Acknowledgements. We are thankful to Yasmin Ochoa, Bill Gomez, and Cindy Wilber for their help in the greenhouse and slug collection as well as Ray Von Itter and Dave Wilson for aiding in logistics and maintenance in the greenhouse facility. Thanks as well to Stanford University's Undergraduate Advising and Research and the Sherwood Fund for the Major Grant that supported IN. Additional funds to support this research were also provided by Stanford University's School of Humanities and Sciences through the Dirzo Lab. We would also like to thank the two anonymous reviewers for providing constructive comments.

Edited by: Daniel Montesinos

Reviewed by: two anonymous referees

\section{References}

Agrawal, A. A.: Induced responses to herbivory and increased plant performance, Science, 279, 1201-1202, https://doi.org/10.1126/science.279.5354.1201, 1998.

Agrawal, A. A.: Induced responses to herbivory in wild radish: effects on several herbivores and plant fitness, Ecology, 80, 1713-1723, https://doi.org/10.1890/00129658(1999)080[1713:IRTHIW]2.0.CO;2, 1999.

Agrawal, A. A.: Specificity of induced resistance in wild radish: causes and consequences for two specialist and two generalist caterpillars, Oikos, 89, 493-500, https://doi.org/10.1034/j.16000706.2000.890308.x, 2000.

Agrawal, A. A.: Transgenerational consequences of plant responses to herbivory: an adaptive maternal effect?, Am. Nat., 157, 555569, 2001.

Agrawal, A. A.: Herbivory and maternal effects: mechanisms and consequences of transgenerational induced plant resistance, Ecology, 83, 3408-3415, https://doi.org/10.1890/00129658(2002)083[3408:HAMEMA]2.0.CO;2, 2002.

Agrawal, A. A. and Kurashige, N. S.: A role for isothiocyanates in plant resistance against the specialist herbivore Pieris rapae, J. Chem. Ecol., 29, 1403-1415, https://doi.org/10.1023/A:1024265420375, 2003.

Agrawal, A. A., Laforsch, C., and Tollrian, R.: Transgenerational induction of defences in animals and plants, Nature, 401, 60-63, https://doi.org/10.1038/43425, 1999a.

Agrawal, A. A., Strauss, S. Y., and Stout, M. J.: Costs of induced responses and tolerance to herbivory in male and female fitness components of wild radish, Evolution, 53, 1093-1104, $1999 \mathrm{~b}$.

Ali, J. G. and Agrawal, A. A.: Specialist versus generalist insect herbivores and plant defense, Trends Plant Sci., 17, 293-302, https://doi.org/10.1016/J.TPLANTS.2012.02.006, 2012.

Bonduriansky, R., Crean, A. J., and Day, T.: The implications of nongenetic inheritance for evolution in changing environ- 
ments, Evol. Appl., 5, 192-201, https://doi.org/10.1111/j.17524571.2011.00213.x, 2012.

Bossdorf, O., Schröder, S., Prati, D., and Auge, H.: Palatability and tolerance to simulated herbivory in native and introduced populations of Alliaria petiolata (Brassicaceae), Am. J. Bot., 91, 856862, https://doi.org/10.3732/ajb.91.6.856, 2004.

Bossdorf, O., Richards, C. L., and Pigliucci, M.: Epigenetics for ecologists, Ecol. Lett., 11, 106-115, https://doi.org/10.1111/j.1461-0248.2007.01130.x, 2008.

Brian Traw, M. and Dawson, T. E.: Reduced performance of two specialist herbivores (Lepidoptera: Pieridae, Coleoptera: Chrysomelidae) on new leaves of damaged black mustard plants, Environ. Entomol., 31, 714-722, https://doi.org/10.1603/0046225X-31.4.714, 2002.

Cipollini, D., Purrington, C. B., and Bergelson, J.: Costs of induced responses in plants, Basic Appl. Ecol., 4, 79-89, https://doi.org/10.1078/1439-1791-00134, 2003.

Cornell, H. V. and Hawkins, B. A.: Herbivore responses to plant secondary compounds: a test of phytochemical coevolution theory, Am. Nat., 161, 507-522, 2003.

Dirzo, R.: Experimental studies on slug-plant interactions: The acceptability of thirty plant species to the slug Agriolimax caruaneae, J. Ecol., 68, 981-998, 1980.

Ehrlich, P. R. and Raven, P. H.: Butterflies and plants: a study in coevolution, Evolution, 18, 586-608, 1964.

Galloway, L. F. and Etterson, J. R.: Transgenerational plasticity is adaptive in the wild, Science, 318, 1134-1136, https://doi.org/10.1126/science.1148766, 2007.

Harvell, D. C.: The ecology and evolution of inducible defenses, Q. Rev. Biol., 65, 323-340, 1990.

Heil, M.: Fitness costs of induced resistance: emerging experimental support for a slippery concept, Trends Plant Sci., 7, 61-67, https://doi.org/10.1016/S1360-1385(01)02186-0, 2002.

Heil, M.: Indirect defence via tritrophic interactions, New Phytol., 178, 41-61, https://doi.org/10.1111/j.1469-8137.2007.02330.x, 2008.

Holeski, L. M., Jander, G., and Agrawal, A. A.: Transgenerational defense induction and epigenetic inheritance in plants, Trends Ecol. Evol., 27, 618-626, https://doi.org/10.1016/j.tree.2012.07.011, 2012.

Holm, L.: World Weeds: Natural Histories and Distribution, John Wiley and Sons, New York, New York, USA, 1997.

Jablonka, E. and Lamb, M. J.: Epigenetic inheritance in evolution, J. Evolution. Biol., 11, 159-183, https://doi.org/10.1046/j.14209101.1998.11020159.x, 1998.

Karban, R. and Nagasaka, K.: Are defenses of wild radish populations well matched with variability and predictability of herbivory?, Evol. Ecol., 18, 283-301, https://doi.org/10.1023/B:EVEC.0000035063.70344.03, 2004.

Karban, R., Agrawal, A. A., Thaler, J. S., and Adler, L. S.: Induced plant responses and information content about risk of herbivory, Trends Ecol. Evol., 14, 443-447, https://doi.org/10.1016/S01695347(99)01678-X, 1999.
Meijden, E.: Plant defence, an evolutionary dilemma: contrasting effects of (specialist and generalist) herbivores and natural enemies, Entomol. Exp. Appl., 80, 307-310, https://doi.org/10.1111/j.1570-7458.1996.tb00941.x, 1996.

Neylan, I., Sobral, M., and Dirzo, R.: Transgenerational induction of wild radish palatability trials results, https://doi.org/10.6084/m9.figshare.5956666.v1, 2018.

O’Neal, M. E., Landis, D. A., and Isaacs R.: An inexpensive, accurate method for measuring leaf area and defoliation through digital image analysis, J. Econ. Entomol., 95, 1190-1194, https://doi.org/10.1603/0022-0493-95.6.1190, 2002.

Poelman, E. H., Galiart, R. J. F. H., Raaijmakers, C. E., van Loon, J. J. A., and van Dam, N. M.: Performance of specialist and generalist herbivores feeding on cabbage cultivars is not explained by glucosinolate profiles, Entomol. Exp. Appl., 127, 218-228, https://doi.org/10.1111/j.1570-7458.2008.00700.x, 2008.

Rasmann, S., De Vos, M., Casteel, C. L., Tian, D., Halitschke, R., Sun, J. Y., Agrawal, A. A., Felton, G. W., and Jander, G.: Herbivory in the previous generation primes plants for enhanced insect resistance, Plant Physiol., 158, 854-863, https://doi.org/10.1104/pp.111.187831, 2012.

Reymond, P., Weber, H., Damond, M., Farmer, E. E., Dicke, M., and Farmer, E. E.: Differential gene expression in response to mechanical wounding and insect feeding in Arabidopsis, The Plant Cell Online, 12, 707-720, https://doi.org/10.1105/tpc.12.5.707, 2000.

Richards, E. J.: Inherited epigenetic variation-revisiting soft inheritance, Nat. Rev. Genet., 7, 395-401, https://doi.org/10.1038/nrg1834, 2006.

Simms, E. L. and Rausher, M. D.: Costs and benefits of plant resistance to herbivory, Am. Nat., 130, 570-581, 1987.

Strauss, S. Y. and Agrawal, A. A.: The ecology and evolution of plant tolerance to herbivory, Trends Ecol. Evol., 14, 179-185, https://doi.org/10.1016/S0169-5347(98)01576-6, 1999.

Takeda, S. and Paszkowski, J.: DNA methylation and epigenetic inheritance during plant gametogenesis, Chromosoma, 115, 27-35, https://doi.org/10.1007/s00412-005-0031-7, 2006.

Thompson, J. N. and Burdon, J. J.: Gene-for-gene coevolution between plants and parasites, Nature, 360, 121-125, https://doi.org/10.1038/360121a0, 1992.

Uller, T.: Developmental plasticity and the evolution of parental effects, Trends Ecol. Evol., 23, 432-438, https://doi.org/10.1016/j.tree.2008.04.005, 2008.

Verhoeven, K. J. F., Jansen, J. J., van Dijk, P. J., and Biere, A.: Stress-induced DNA methylation changes and their heritability in asexual dandelions, New Phytol., 185, 1108-18, https://doi.org/10.1111/j.1469-8137.2009.03121.x, 2010.

Zangerl, A. R.: Evolution of induced plant responses to herbivores, Basic Appl. Ecol., 4, 91-103, https://doi.org/10.1078/14391791-00135, 2003. 